\title{
Intra-Abdominal Leiomyosarcoma in a Ferret (Mustela Putorius Furo): Histopathological and Immunohistochemical Characterization
}

\author{
Marzia PEZZOLATO ${ }^{1) *}$, Katia VARELLO ${ }^{1)}$, Daniela MASCARINO ${ }^{1)}$, Elisa BOZZATO2), \\ Marta VASCELLARI ${ }^{2)}$ and Elena BOZZETTA ${ }^{1)}$ \\ ${ }^{1)}$ Istituto Zooprofilattico Sperimentale del Piemonte, Liguria e Valle d'Aosta - Area Neuroscienze e Genetica- Laboratorio di \\ Immunoistopatologia, Via Bologna 148, 10154 Torino and ${ }^{2)}$ Istituto Zooprofilattico Sperimentale delle Venezie, Laboratorio \\ Istopatologia, Viale dell'Università 10, 35020 Legnaro- Padova- Italy
}

(Received 26 June 2007/Accepted 26 December 2007)

ABSTRACT. A 5 years old female ferret with an abdominal palpable mass confirmed at echo-graphic examination died during an explorative laparotomy. A single lymph-node-like nodule was found adjacent to the intestinal loops. The round mass well circumscribed, solid and white, histologically, at low magnification, appeared encapsulated and built up by a population of atypical spindle cells arranged in interwoven fascicles. The cells had high anisocytosis degree, moderate mitotic activity and prominent nucleoli. A central area of necrosis was present. To characterise the tumour immunohistochemically cytokeratin, vimentin, S-100, melan-A, vWF, desmin, actin and $\alpha$-actin were applied. Neoplastic cells resulted positive to vimentin, actin and $\alpha$-actin. Based on the histological and immunohistological pattern a diagnosis of leiomyosarcoma was made.

KEY WORDS: abdomen, ferret, leiomyosarcoma.

In the last years there has been a remarkable increase in the number of documented neoplasms found in ferrets and among these soft tissue sarcomas are reported and characterized less frequently than carcinomas and round cell tumours $[4,5]$. Among the soft tissue tumors the muscular ones previously described include a large number of dermal leiomyosarcoma [3], one rhabdomyosarcoma of the lateral thorax [1], one leiomyosarcoma free floating in the abdomen [2] and a large number of leiomyosarcoma of the female reproductive tract [1].

This short communication describes a case of intraabdominal leiomyosarcoma in a domestic pet ferret of undetermined origin.

A 5 years old female ferret with lethargy, alopecia and hypothermia was conducted to veterinary examination: the ferret was depressed and had an abdominal palpable mass confirmed at echo-graphic examination. During an explorative laparotomy a single unattached nodule was found floating freely in the abdominal cavity adjacent the intestinal loops. Following the death of the animal, occurred during the surgery, the single lymph-node-like abdominal mass was identified, biopsied, fixed in 10 per cent neutral-buffered formalin and sent to the Histopathology laboratory of Istituto Zooprofilattico Sperimentale of Torino.

At macroscopic examination the mass was $1 \mathrm{~cm}$ in diameter and appeared round, well-demarcated, solid and white.

The tissue was trimmed, routinely processed, embedded in paraffin wax, sectioned at 3-5 $\mu \mathrm{m}$ and stained with haematoxylin and eosin (HE) and histochemically with Mas-

\footnotetext{
* Correspondence to: Pezzolato, M., Istituto Zooprofilattico Sperimentale del Piemonte, Liguria e Valle d'Aosta - Area Neuroscienze e Genetica- Laboratorio di Immunoistopatologia, Via Bologna 148, 10154 Torino- Italy.

e-mail: istopatologia.ufficio@izsto.it
}
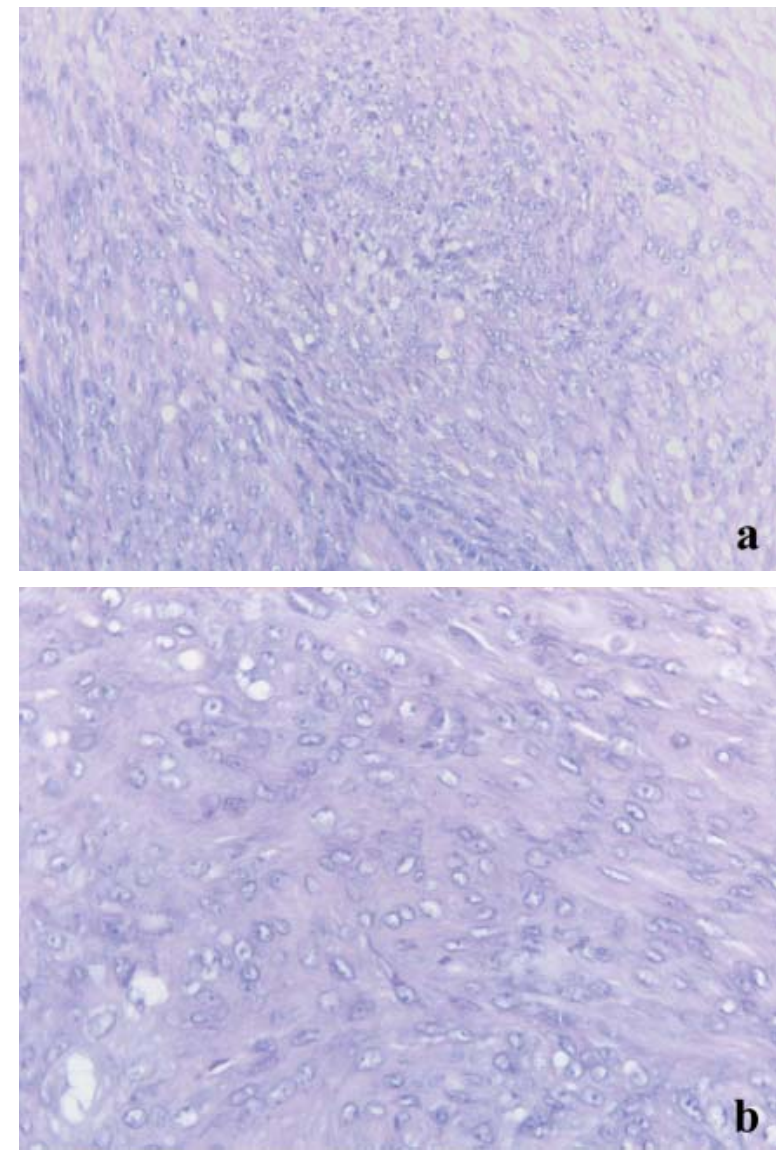

Fig. 1. (a) Interlacing fascicles of densely packed spindle cells $($ HE $20 \times)$; (b) $($ HE $40 \times)$. 


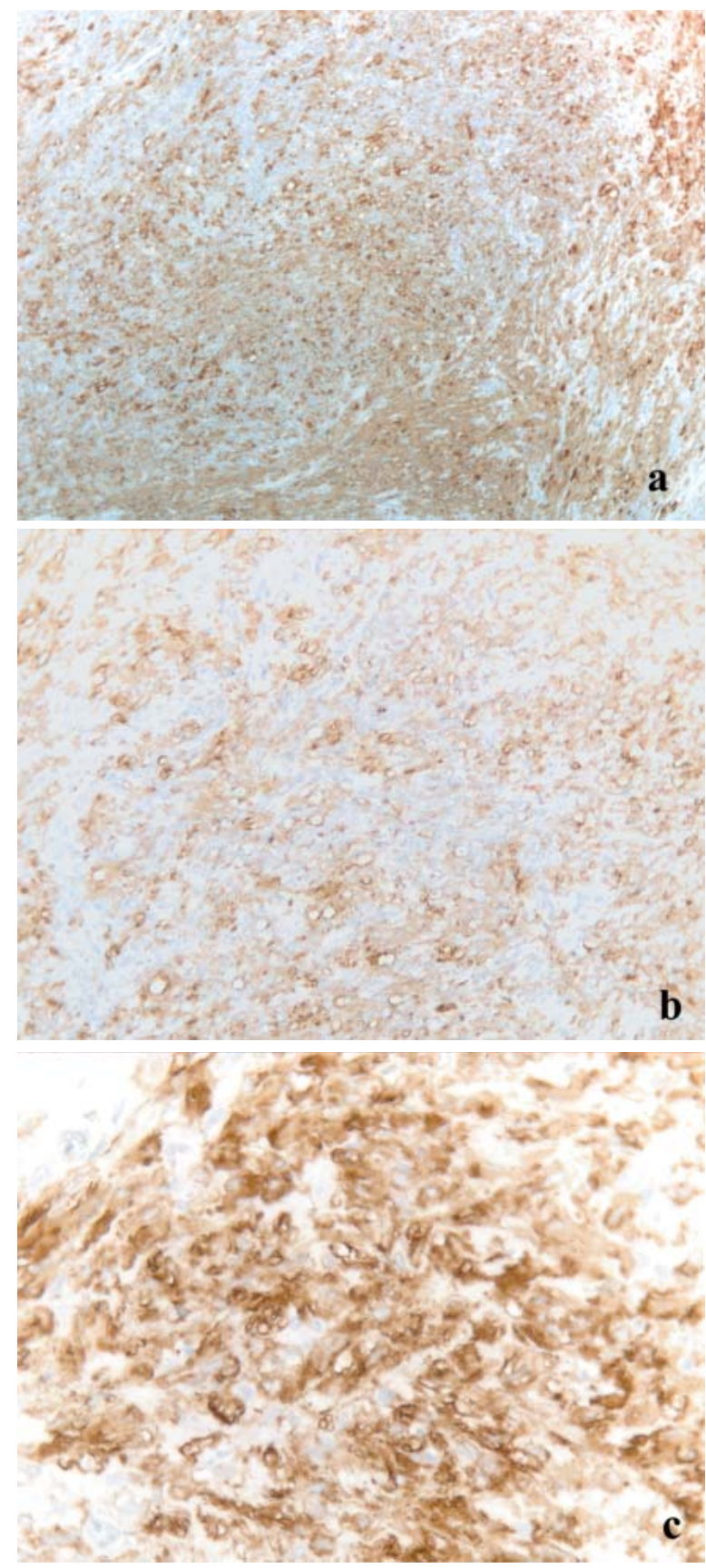

Fig. 2. (a) Dense intracytoplasmic expression of $\alpha$-smooth muscle actin $($ IHC $10 \times)$; (b) $($ IHC $20 \times)$; (c) $($ IHC $40 \times)$.

son's trichrome.

Histologically, at low magnification, the neoplasm appeared encapsulated but well-demarcated. It was built up by a population of atypical spindle cells arranged in interwoven fascicles supported by a small amount of fibrovascular stroma. Neoplastic cells had a moderate amount of acidophilic cytoplasm that was delicately fibrillar. Nuclei were central, oval to elongated with clumped chromatin and
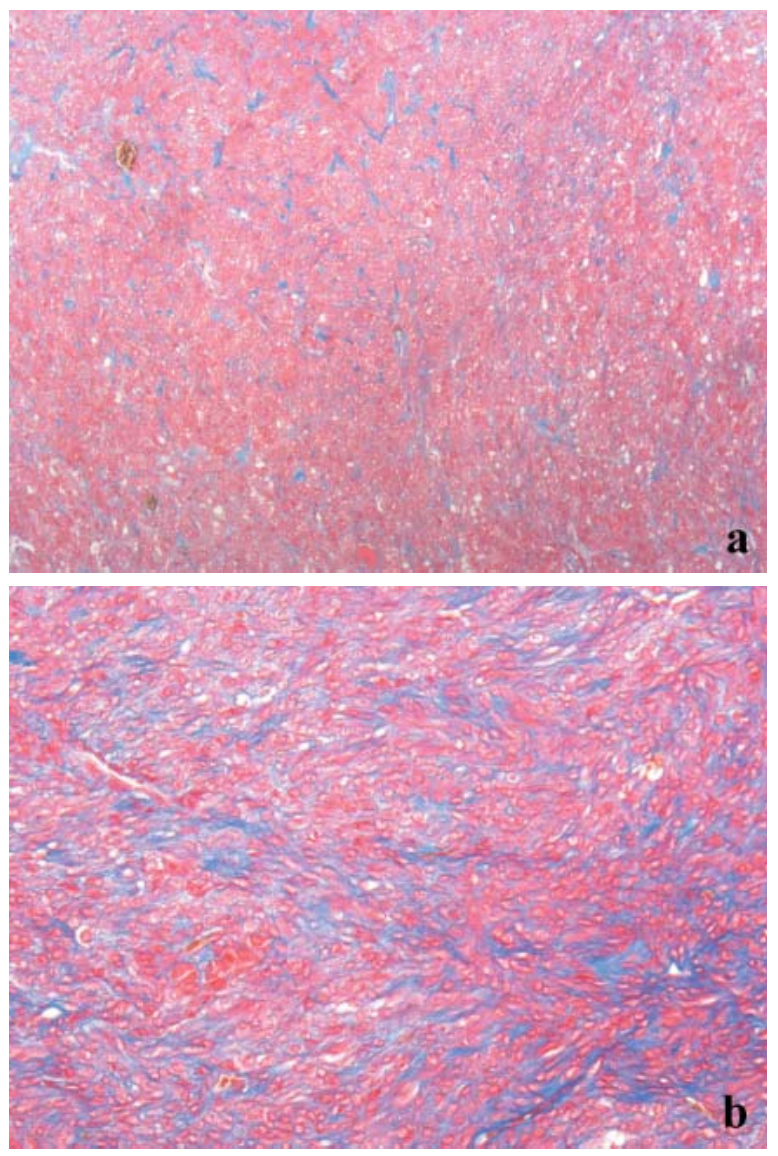

Fig. 3. (a) Scanty presence of collagen fibers (Masson's Trichrome staining $10 \times$ ); (b) (Masson's Trichrome staining $20 \times)$.

1 or 2 prominent basophilic nucleoli. Mitotic index averaged from 3 to 7 mitoses per $10 \mathrm{HPF}$ (high power fields). A central area of necrosis was present (Fig. 1).

Histochemically the cells appeared almost all red and only few collagen fibres could be identified in blue (Fig. 3).

The HE histological staining didn't allow to formulate a definitive diagnosis so immunohistochemical analysis was performed to differentiate among intra-abdominal spindle cell lesions: fibromatosis, various sarcomas-in particular, leiomyosarcoma, liposarcoma, and malignant peripheral nerve sheath tumour.

To characterise the tumour immunohistochemically cytokeratin (Dako, monoclonal, heat-induced epitope retrieval high $\mathrm{pH}, 1: 50$ ), vimentin (Dako, monoclonal, heatinduced epitope retrieval high $\mathrm{pH}, 1: 100), \mathrm{S}-100$ (Dako, polyclonal), melan-A (Novocastra, monoclonal, heatinduced epitope retrieval pH 6, 1:25), vWF (Dako, polyclonal, proteinase $\mathrm{K}$ pre-treatment, 1:600) desmin (Dako, monoclonal, trypsinization for $30 \mathrm{~min}$ at $37^{\circ} \mathrm{C}, 1: 50$ ), muscle specific actin (Dako, monoclonal, 1:50) and $\alpha$-smooth muscle actin (Dako, monoclonal, trypsinization for $30 \mathrm{~min}$ at $\left.37^{\circ} \mathrm{C}, 1: 50\right)$ were performed on selected sections. Posi- 
tive immunohistochemical controls included appropriate cells of normal ferret organ with all primary antibodies evaluated.

Neoplastic cells resulted negative to cytokeratin, melanA, factor VIII, S-100, and desmin, while were positive to vimentin, muscle specific actin and $\alpha$-actin in the whole tissue (Fig. 2).

These results indicated that the proliferated cells were of smooth muscle origin. Based on the histological pattern and on the immunohistological findings it was possible to formulate a diagnosis of leiomyosarcoma. The primary site couldn't be determined, as its origin from genital, digestive or urinary system couldn't be confirmed at gross anatomical pathological examination.

The occurrence of abdominal leiomyosarcoma is rare in animal and a single case of leiomyosarcoma found unattached in the abdominal cavity was reported until now in a female ferret by American authors [2].

\section{REFERENCES}

1. Brunnert, S. R., Herron, A. J. and Altman, N. H. 1990. Lab. Anim. Sci. Mar. 40: 208-210.

2. Dillberger, J. E. and Altman, N. H. 1989. J. Comp. Pathol. Feb. 100: 161-176.

3. Mikaelian, I. and Garner, M. M. 2002. J. Vet. Diagn. Invest. May. 14: 262-265.

4. Rickman, B. H., Craig, L. E. and Goldschmidt, M. H. 2001. Vet. Pathol. 38: 710-711.

5. Zaias, J., Kornegay, R. W., Altman, N. H. and Herron, A. J. 2001. Contemp. Top. Lab. Anim. Sci. Jan. 40: 43-44. 